\title{
A GENERAL THEOREM CONCERNING ABSOLUTELY CONVERGENT SERIES
}

\author{
$B y$ G. H. HaRDY.
}

[Received October 9th, 1903.-Read November 12th, 1903.]

1. My object in this paper is to prove a theorem which contains as special cases a number of well known theorems concerning absolutely convergent series. Among these are, for instance, the following theorems :-

(i.) the terms of an absolutely convergent simple series may be permuted in any manner withont altering the sum of the series;

(ii.) an absolutely convergent double series may be rearranged in any manner as a simple series;

(iii.) an absolutely convergent double series may be summed indifferently by rows or by columns (Cauchy's double-series theorem).

It occurred to me recently that all these theorems are very particular cases of a general theorem concerning the rearrangement of series. This theorem not only includes all the known theorems, but is, as I shall show, the most general possible theorem of its lind; and, in my opinion, its introduction lends to the theory a simplicity and generality which is otherwise lacking.

\section{Series of Type $\beta . *$}

2. The ordinary simply infinite series is of type $\omega$, that is to say, its "ordinal type" is that of the class of numbers $<\omega$. The sum of two infinite series $a_{0}+a_{1}+\ldots+b_{0}+b_{1}+\ldots$ is of type $\omega .2 . \quad A$ doubly infinite series, summed by rows or columns, is of type $\omega^{2}$; summed by diagonals, it becomes of type $\omega$. As the class of numbers $<\beta$ is enumerable, it is clear that we can arrange any enumerably infinite set of terms in type $\beta$.

* In what follows I shall denote by Greek letters $a, \beta, \gamma, \ldots$ numbers of Cantor's firs! ('n!l second classen $0,1,2, \ldots, \omega, \omega+1, \ldots, \omega .2, \ldots, \omega^{*}, \ldots, \omega^{\omega}, \ldots$. When it is necessary to distinguish specially the finite numbers (numbers of the first class) $\mathrm{I}$ shall use $m, n, n, \ldots$. 
Suppose then that we have a series of type $\beta$, and, for the present, that its terms $u_{0}, u_{1}, \ldots, u_{\omega}, \ldots, u_{\omega .2}, \ldots, u_{\omega^{2}}, \ldots, u_{\omega^{\alpha}}, \ldots$ are all $\geqslant 0$. There may or may not be a last term.

Sum of a Series of Type $\beta$.

3. Since all the $u$ 's are positive,

$$
s_{n}=u_{0}+u_{1}+\ldots+u_{n-1}=\sum_{\gamma<n} u_{\gamma}
$$

tends to a limit for $n=\infty$. We call this $s_{\omega}$ or $\Sigma u_{\gamma}$. Of course it may be that $s_{\omega}=\infty$. If $s_{\omega}$ is finite, $s_{\omega+n}=s_{\omega}+u_{\omega}+\ldots+u_{\omega+n-1}$ has a limit for $n=\infty$, which we call $s_{\omega .2}$. This again may $=\infty$, and, if $s_{\omega}=\infty$, we regard $s_{\omega .2}$ as also $=\infty$. Thus we may define $s_{\omega . n}$ for any value of $n$, and it is clear that $s_{\omega} \leqslant s_{\omega .2} \leqslant s_{\omega .3} \ldots$; so that $\lim _{n=\infty} s_{\omega . n}$ is determinate. We call this $s_{\omega^{2}}$. It is clear that we may proceed thus and define $s_{a}$ for all values of $a \leqslant \beta$. We call $s_{\beta}$ the sum of the series. If any $s_{a}=\infty$, we agree that $s_{a^{\prime}}=\infty\left(a \leqslant \alpha^{\prime} \leqslant \beta\right)$. In this case we say that the series is divergent. If $s_{\beta}$ is finite, we say that the series is convergent in type $\beta$, and write $s_{\beta}=\sum_{\gamma<\beta} u_{\gamma}$.

The object of this paper is to prove the following

THEOREM.-If a series is absolutely convergent in type $\beta$, it remains absolutely convergent when its terms are rearranged in another type $\beta^{\prime}$, and its sums in the two types are the same.

I may observe that the theorem covers the case of any rearrangement of the series in the same type.

\section{Elementary Properties of Series of Type $\beta$.}

4. The series thus defined possess some of the characteristic properties of ordinary series. Thus it is easy to prove that

$$
\text { (1) } \sum_{\gamma<\beta}\left(u_{\gamma}+v_{\gamma}\right)=\sum_{\gamma<\beta} u_{\gamma}+\sum_{\gamma<\beta} v_{\gamma}
$$

if $\Sigma u_{\gamma}, \Sigma v_{\gamma}$ are convergent, and

$$
\text { (2) } \sum_{\gamma<\beta} \kappa u_{\gamma}=\kappa \sum_{\gamma<\beta} u_{\gamma} \text {. }
$$


1903.] A general theorem CONCERNing abSOLUteLX CONVERgENT SERIES. 287

These propositions follow readily from the equations

and

by induction.*

$$
\text { (a) } \sum_{\gamma<a+1} u_{\gamma}=\sum_{\gamma<a} u_{\gamma}+u_{a}
$$

Again,

(3) If $\sum_{\gamma<\beta} u_{\gamma}$ is convergent, and $v_{\gamma} \leqslant u_{\gamma}$ for all values of $\gamma$, then $\Sigma v_{\gamma}$ is convergent, and its sum $\leqslant s_{\beta}$.

The proofs of these theorems are so simple that I need hardly write them out at length. In each case we show by means of the equations (a) and (b) that (i.), if they hold for series of type $a$, they hold for series of type $a+1$; and (ii.) that, if they hold for series of types $a_{1}, a_{2}, \ldots$ $\left(a_{1}<a_{2}<\ldots, \lim \alpha_{n}=\alpha\right)$, they hold for series of type $a$. As they evidently hold for series containing only one term, they hold generally.

\section{Proof of the Theorem.}

5. In order to prove the theorem of $\$ 3$ we must establish a series of lemmas.

Lemma 1.-If a convergent series of type $\beta$ is arranged in type $\omega$, the new series is convergent and its sum $\leqslant s_{\beta}$.

For the sum of any finite number of terms of the series $\leqslant s_{\beta}$. This follows from (3), $\$ 4$, if we replace all the other terms of the series by zeros. The lemma follows immediately.

Lemma 2.-If a series is convergent in type $\beta$, we can find a finite number of terms whose sum $>s_{\beta}-\delta$, where $\delta$ is an arbitrarily small positive quantity; and, if it is divergent, we can find a finite number of terms whose sum $>G$, an arbitrarily large quantity.

For suppose the first part true for series of type $a$. Then it is true for series of type $a+1$. For, if $u_{\gamma_{1}}+u_{\gamma_{2}}+\ldots+u_{\gamma_{N}}>s_{a}-\delta$,

$$
u_{\gamma_{1}}+u_{\gamma_{2}}+\ldots+u_{\gamma_{X}}+u_{a+1}>s_{a+1}-\delta \text {. }
$$

Again, suppose the theorem true for series of the types $a_{1}, a_{2}, \ldots$, and $a=\lim _{n=\infty} \alpha_{n}$. Then, since $s_{a}=\lim _{n=\infty} s_{a_{u}}$, we can choose $n$ so that

$$
s_{a_{n}}>s_{a}-\frac{1}{2} \delta \text {. }
$$

- One fundamental property of an ordinary series which is obviously not possessed by the generalized series is that in an ordinary series we can assign a rank after which the terms are as small as we please. 
But we can choose $N$ numbers $\gamma_{1}, \ldots, \gamma_{N}<a_{n}$ so that

$$
u_{\gamma_{1}}+\ldots+u_{\gamma_{y}}>s_{a_{n}}-\frac{3}{2} \delta>s_{a_{n}}-\delta \text {. }
$$

As the first part of the lemma is true for series of one term, it is true generally. A similar proof applies to the second part.

Lema 3.-If a series is convergent in type $\omega$ and its sum is $s_{\omega}$, it vill be convergent when arranged in type $\beta$ and its sum will be $\leqslant s_{\omega}$.

This follows at once by a redluctio ad absurdum from Lemma 2.

Lema 4.-If a series is convergent in type $\beta$, it will be convergent when arranged in type $\omega$, and the sums will be the same, and conversely.

Suppose it convergent in type $\beta$. By Lemma 1 it is convergent in type $\omega$, and its sum $\leqslant s_{\beta}$. But, if its sum $<s_{\beta}$, it follows from Lemma 3 that its sum in type $\beta<s_{\beta}$, which is absurd. The converse is proved similarly. From Lemma 4 the theorem follows at once.

A well known particular case of the theorem is the theorem concerning the summation of a doubly infinite series ly rows, columns, or diagonals. In this case we have to deal with two arrangements in type $\omega^{2}$ and one in type $\omega$. Similar theorems for multiple series of any order follow at once.

[It is perhaps worth while at this point to emphasize the distinction between a multiple and a repeated series, as it is only recently that the notion of a multiple series has been made at all precise. According to Joldan the double series $\sum_{m, n=1}^{\infty} u_{m, n}$ is convergent only if all possible simply infinite series formed out of it are convergent. According to Pringsheim and Stolz it is convergent if certain large class of them are. The point of importance for my present purpose is that in any case to assert the convergence of a double or multiple series is not really to assert the convergence of one series of a type different from that of the ordinary simple series, but to assert the convergence of a whole class of series of the ordinary type. But to assert the convergence of a reperted series is to assert the convergence of one series of a type in any case $>\omega$. The theorem applies to either kind of series.]

6. The theorem is easily extended to any absolutely convergent series, real or complex.

An absolutely convergent series of type $\beta$ is a series of type $\beta$ which is convergent when every term is replaced by its modulus. In the first place it is to he observerl that the definitions and explanations of $\$ 3$ were 
given only for series of positive and zero terms. They apply, however, to series of arbitrary terms, except that when the terms are not all positive we cannot be sure that a definite limit (finite or infinite) will be found at each stage of the process. However, we can prove that an absolutely convergent series of type $\beta$ is convergent; that is to say, a definite limit $s_{\beta}$ results if we carry out the process of $\S 3$.

For suppose first that the terms are all real, and define two new series $\Sigma v_{\gamma}, \Sigma w_{\gamma}$ by the equations

$$
v_{\gamma}=u_{\gamma}\left(u_{\gamma}>0\right)=0\left(u_{\gamma}<0\right), \quad v_{\gamma}=0\left(u_{\gamma}>0\right)=-u_{\gamma}\left(u_{\gamma}<0\right) ;
$$

then $u_{\gamma}=v_{\gamma}-v_{\gamma}$. Also, since $\left|v_{\gamma}\right| \leqslant\left|u_{\gamma}\right|, v_{\gamma} \leqslant\left|u_{\gamma}\right|, \quad \Sigma v_{\gamma}, \Sigma v_{\gamma}$ are convergent. Moreover, from (1) of $\S 4, \sum_{\gamma<a} u_{\gamma}$ is convergent and

$$
\sum_{\gamma<a} u_{\gamma}=\sum_{\gamma<a} v_{\gamma}-\sum_{\gamma<a} w_{\gamma}
$$

Now apply the theorem of the last paragraph to each of these series separately. We find that $\Sigma u_{\gamma}=\Sigma^{\prime} v_{\gamma}-\Sigma^{\prime} v_{\gamma}, \Sigma^{\prime}$ denoting a series of the new type. And the right-hand is $\Sigma^{\prime} u_{\gamma}$.

Next suppose the terms $u_{\gamma}$ complex, and let $u_{\gamma}=v_{\gamma}+i w_{\gamma}$. Then, since $\left|v_{\gamma}\right| \leqslant\left|u_{\gamma}\right|,\left|w_{\gamma}\right| \leqslant\left|u_{\gamma}\right|$, the series $\Sigma v_{\gamma}, \Sigma w_{\gamma}$ are absolutely convergent; and the theorem follows as before. The theorem is therefore proved for all absolutely convergent series.

7. It only remains for me to justify the assertion that this theorem is the most general possible theorem of its kind. This is evident if we consider that any rearrangement of an ordinary series of type $\omega$ must lead to an arrangement in some type $\beta$. The possibility of a series with a nonenumerable infinity of positive terms is excluded by Cantor's theorem that any set of intervals on a straight line must be enumerable.*

8. In conclusion, I give an example, suggested by a passage in Schoenflies's Mengenlehre, of an arrangement of the series

$$
\frac{1}{1^{2}}+\frac{1}{2^{2}}+\frac{1}{3^{2}}+\ldots
$$

"See e.g., W. H. Young, "On Sets of Intervals," Proc. London Math. Soc., Vol. xxxv., p. 248. Another question is whether it is possible to define the sum of a series with a nonenumerable number of terms which are not all positive. It seems to me that the answer is probably no; but, as the question involves considerations of some logical difficulty, I shall not at present attempt to justify this answer.

SER. 2. VOL. 1. No. 838 . 
290 A GENERAL THEOREM CONCERNING ABSOLUTELLY CONVERGENT SERIES.[Nov.12, in type $\beta$, where $\beta$ is at any rate $>\omega^{\omega}$. It is

$$
\begin{aligned}
& \quad \frac{1}{1^{2}}+\frac{1}{2^{2}}+\frac{1}{3^{2}}+\frac{1}{5^{2}}+\frac{1}{7^{2}}+\frac{1}{11^{2}}+\ldots \\
& +\frac{1}{2^{2} \cdot 3^{2}}+\frac{1}{2^{2} \cdot 5^{2}}+\frac{1}{2^{2} \cdot 7^{2}}+\ldots \\
& +\frac{1}{3^{2} \cdot 5^{2}}+\frac{1}{3^{2} \cdot 7^{2}}+\ldots \\
& +\frac{1}{5^{2} \cdot 7^{2}}+\ldots \\
& +\ldots \\
& +\frac{1}{2^{2} \cdot 3^{2} \cdot 5^{2}}+\frac{1}{2^{2} \cdot 3^{2} \cdot 7^{2}}+\frac{1}{2^{2} \cdot 3^{2} \cdot 11^{2}}+\ldots \\
& +\frac{1}{2^{2} \cdot 5^{2} \cdot 7^{2}}+\frac{1}{2^{2} \cdot 5^{2} \cdot 11^{2}}+\ldots \\
& +\frac{1}{2^{2} \cdot 7^{2} \cdot 11^{2}}+\ldots \\
& +\ldots \\
& +\frac{1}{3^{2} \cdot 5^{2} \cdot 7^{2}}+\ldots \\
& +\ldots
\end{aligned}
$$

It is easy to see that, if we carry out this scheme systematically, $\sum_{\gamma<\omega^{2}}$ contains every $1 / n^{2}$ for which $n$ is a product of two primes (including 1), $\sum_{\gamma<\omega^{3}}$ includes every $1 / n^{2}$ for which $n$ is a product of three primes, and so on. Hence $\underset{\gamma<\omega^{*}}{\sum}$ includes only those terms for which $n$ is a product of different primes. The complete series (however we go on with it) is therefore of type $\beta>\omega^{\omega}$. 